\title{
Review
}

\section{The importance of ultra-high voltage electron microscopy in materials science}

\author{
By Hiroshi FuJITA, M. J. A. \\ Professor Emeritus of Osaka University \\ (Contributed by Hiroshi FuJITA, M. J. A.)
}

\begin{abstract}
Natural science is now extensively developed thanks to electron microscopy, but around 1950, only thin specimens were used because of the low accelerating voltages. The behavior of crystalline materials is very sensitive to specimen thickness, and thus in Japan a practical $500 \mathrm{kV}$ electron microscope was constructed in 1965 for thicker specimens. It was shown that simultaneous reflection increases with increasing accelerating voltage, so that the maximum observable specimen thickness increases almost linearly up to $500 \mathrm{kV}$. Since simultaneous reflection becomes prominent above 1,500 kV, the in-situ observation of various phenomena representative of most bulk materials has been carried out with an ultra-high voltage electron microscope, whose accelerating voltages can reach 3,000 kV. Thus, even the characteristics of high-Z materials have been clarified in detail, and new applications, such as foreign atom implantation and the formation of non-equilibrium phases, have also been developed. The present account deals mainly with the importance of 3,000 kV electron microscopes, as applied to the new research fields of materials science.
\end{abstract}

Key words: Simultaneous reflection; electron channeling; objective aperture effect; in-situ observation; foreign atom implantation; amorphization.

Introduction. In natural science, there is a "threshold value" above which a new field of research is opened, and transmission electron microscopes (EMs) have opened such a new field. Ever since dislocation images were obtained with $100 \mathrm{kV} \mathrm{EMs}{ }^{1,2)}$ in Europe and with a $50 \mathrm{kV}$ EM in Japan ${ }^{3)}$ independently in 1956-57 by means of different specimen preparation methods, materials science has seen a rapid progress thanks to electron microscopy. Dislocations are the most important lattice defects, but their behavior is very sensitive to the thickness of the crystalline materials. As a result, not only the behavior but also the density of the dislocations changes dramatically when the specimen thickness becomes smaller than the critical value, and the changes depend on the kind of phenomena and materials involved. ${ }^{4)-6)}$ This is one reason why, in order to get the same information about the behavior of lattice defects in thin materials as one would get from bulk materials, highvoltage electron microscopes (HVEMs) are necessary.

\footnotetext{
†) Home address: 5-12-22 Yamatedai, Ibaraki, Osaka 5670009, Japan.
}

Practically, $500 \mathrm{kV}$-class HVEMs are sufficient for this purpose if the materials observed are made of light metals whose atomic number is smaller than $20,{ }^{6,7)}$ but the accelerating voltage must be increased with increasing atomic number of the materials observed. Various phenomena occurring in bulk materials have generally been investigated in-situ with ultra-HVEMs with accelerating voltages at least higher than 2,000 kV (2 MV), and/or with HVEMs with voltages lower than $1,500 \mathrm{kV}$, and the mechanisms behind those phenomena have been made clear dynamically. ${ }^{8)}$

The present report is mainly concerned with the importance of electron channeling at high voltages and with various applications of ultra-HVEMs in materials science.

Voltage dependence of the maximum observable specimen thickness. In 1920-30, the structure sensitivity of the behavior of materials was considered to be closely related to existence of lattice defects, and a dislocation model was first proposed by Yamaguchi as reflecting the most important lattice defect. ${ }^{9)}$ After that, many theories were proposed regarding the dislo- 
cations, and thus acquiring direct evidence showing the existence of dislocations became one of the most important subjects in materials science. Since the wavelength of the electrons emitted by EMs is very short compared with those generated by other means, not only high-resolution imaging but also selected-area electron diffraction from very small regions was expected. Electrons, however, can hardly traverse thick materials, and so only very thin foils could be used in general as the specimens. Furthermore, the functional features of EMs before 1950 were so poor that each individual dislocation could not be discriminated when Heidenreich first tried to observe dislocations in metals. ${ }^{10)}$

As mentioned, the direct observation of dislocations succeeded in 1956, as the functional features of EMs improved. The results were epoch-making, but even 100 $\mathrm{kV}$ EMs were not sufficient to determine the correct density of the dislocations, so that frequently no dislocations were observed even in heavily worked specimens. Of course, in such thin metal foils, the behavior of the material changes dramatically, as will be discussed later. These changes are the result of the behavior of dislocations, which are very sensitive to the specimen thickness because of the heavy lattice distortion around dislocations.

This is one reason why the improvement of EMs became a topic of theoretical discussion; the aim was to be able to observe thicker specimens showing the same behavior as bulk materials. However, the conclusions of those discussions were very pessimistic. ${ }^{8)}$ According to the theory proposed at that time, the penetration power of electrons $(P)$ can be expressed by $1 / \mu_{0}\left(\mu_{0}\right.$ : the absorption coefficient of electrons), which is inversely proportional to $\lambda^{1 / 2}$ ( $\lambda$ : the relativistic wavelength of electrons). ${ }^{11)}$ This meant that the value of $P$ is almost saturated at about $500 \mathrm{kV}$ due to the relativistic effect, and increases only 3.3 times compared to the value at $100 \mathrm{kV}$, even when the accelerating voltage is increased to infinity. ${ }^{6), 10)}$ Since the value of $P$ was defined as the transmission distance of incident electrons until their intensity decreases to $1 / e$, most electron microscopists thought that the value of $P$ corresponds to the maximum observable specimen thickness $\left(t_{M}\right){ }^{6), 8)}$ This hypothesized voltage dependence of $t_{M}$ also seemed to be supported by some experimental data, ${ }^{6), 12), 13)}$ which, however, did not take into account the objective aperture effect, as shown later.

Furthermore, even if the value of $t_{M}$ were to increase at high voltages, other worries surfaced ${ }^{6), 8)}$ : (a) the remarkable image broadening due to the inelastic scattering of incident electrons in thick specimens, ${ }^{14)}$ (b) the heavy electron irradiation damage occurring during EM observation, ${ }^{15)}$ (c) the difficulty of recording images, etc. Also, technical challenges, such as the construction of a stable accelerating tube, mechanical vibration proofing, the construction of a shield against electromagnetic fields from surrounding electric currents and against the X-rays emitted by the EM column, etc., became acute with accelerating voltages of about the third order of magnitude.

In such a generally pessimistic atmosphere, the author and his group at the National Research Institute for Metals (NRIM) constructed in 1963-66 two practical double-tank-system 0.5 MV HVEMs in cooperation with Shimadzu, Ltd., ${ }^{7,16)}$ as the author had already established that at least recrystallization occurs even in thin aluminum foils about $1 \mu \mathrm{m}$ in thickness. ${ }^{3), 17)}$ The newly built 0.5 MV HVEMs consisted of the following two parts: 1) a Cockcroft-Walton circuit for the high-voltage generator, and 2) a cascade-type accelerator with an applied voltage at each electrode of 50 or $25 \mathrm{kV}$ (the gap between adjacent electrodes could be shortened, depending on the voltage setting). The high-voltage generator and the accelerator were kept in two separate tanks, in insulating gases such as $\mathrm{SF}_{6}$ and freon at a pressure of about 3 $\mathrm{kgf} / \mathrm{cm}^{2}$ (0.3 MPa). In 1965, the author established the following as a result of his work with the $0.5 \mathrm{MV}$ HVEMs: (1) the objective aperture angle must be increased with increasing accelerating voltage in order to take advantage of the simultaneous reflections, and then the value of $t_{M}$ increases almost linearly up to 0.5 MV, and (2) in-situ observation of the same phenomena as those in bulk materials becomes possible at 0.5 MV when the atomic number of the constituent atoms of the materials is less than about 20. ${ }^{7)}$ As a consequence of the author's pioneering work, about 60 HVEMs and ultraHVEMs have been installed, not only in Japan ${ }^{13), 15)}$ but in other countries as well, ${ }^{18)}$ as shown in the Appendix.

Based on the aforementioned results, the author concluded that in-situ observation of the same phenomena as those in almost all kinds of bulk materials becomes possible with a 3 MV ultra-HVEM ${ }^{19}$; he planned the construction of a 3 MV-class double-tanksystem ultra-HVEM in 1966, when the NRIM decided to move to the Tsukuba area. After that, the author moved to Osaka University in 1967. At that time, there was an ongoing project to construct a 1 MV HVEM of the superconducting linear-accelerator type at Osaka University, but the author's plan gained prominence because of his extensive practical experience. And so, his 


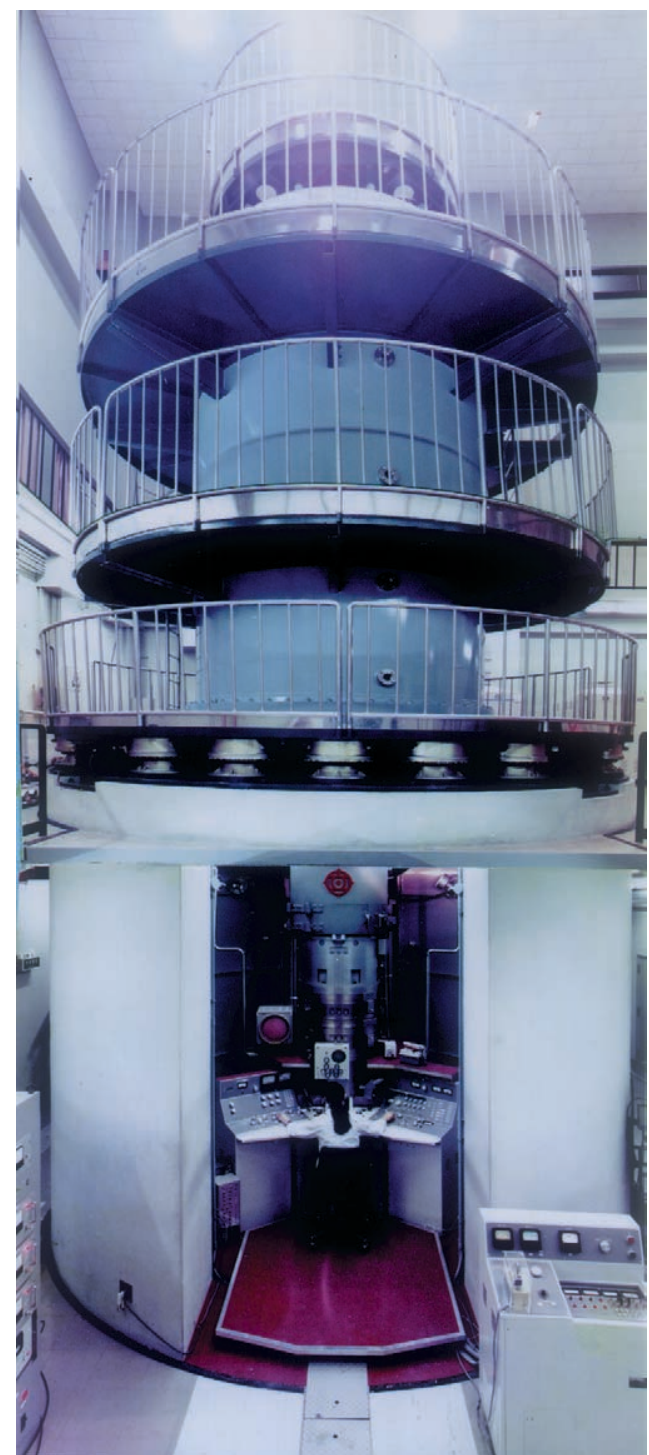

Fig. 1. Outside view of a 3 MV ultra-HVEM at Osaka University.

group installed a $3 \mathrm{MV}$ tank-in-tank ultra-HVEM at Osaka University in 1970 with the cooperation of Hitachi, Ltd., as shown in Fig. 1. ${ }^{8), 19), 20)}$ In 1972, they succeeded in examining the voltage dependence of $t_{M}$ up to $3 \mathrm{MV}$ for various materials, thus finally demonstrating the validity of the author's conclusion. ${ }^{21), 22)}$ The ultra-HVEM was further improved to become remote-controlled from a separate room, ${ }^{23)}$ and is still working at $2 \mathrm{MV}$ at present. A new 3.5 MV ultra-HVEM with advanced functional features was thereafter installed at Osaka University $^{24)}$ based on the technical data obtained with the first $3 \mathrm{MV}$ ultra-HVEM.

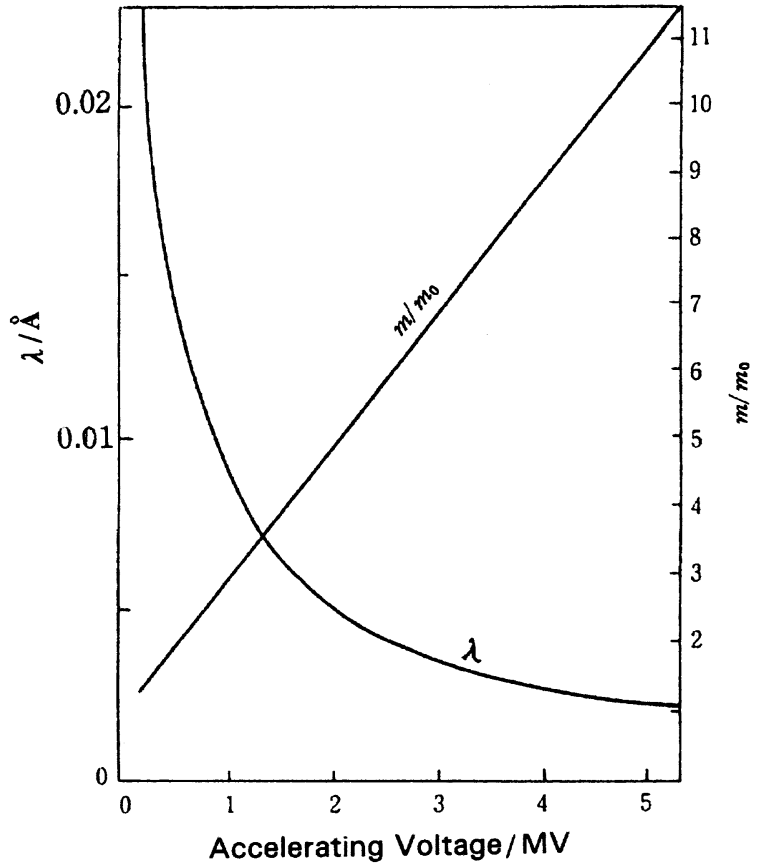

Fig. 2. Relativistic effects on the wavelength $(\boldsymbol{\lambda})$ and the mass $(m)$ of an electron ( $m_{0}$ : static mass of an electron).

Effects of electron channeling and the objective aperture angle on the value of $\boldsymbol{t}_{\boldsymbol{M}}$. Both effects become prominent at high voltages because of strong excitation of the simultaneous reflection.

Voltage dependence of the simultaneous reflection. The aforementioned hypothesis regarding the voltage dependence of $t_{M}$ was thought to hinge on the transmissive power, which is inversely proportional to $\lambda^{1 / 2}$. This meant that the value of $t_{M}$ would be almost saturated at $0.5 \mathrm{MV}$, as expected from the voltage dependence of $\lambda$ shown in Fig. 2. Practically, however, the degree of simultaneous excitation of many waves markedly increases with increasing accelerating voltage. Figures $3 \mathrm{a}$ and $\mathrm{b}$ show such a phenomenon appearing in a pair of $\{111\}$ systematic-reflection bend contours in wedge-shaped aluminum specimens ${ }^{22), 25)}$; micrographs a and b were taken at 0.5 and $3 \mathrm{MV}$ respectively. At $0.5 \mathrm{MV}$, the symmetry position of the $\{111\}$ reflections becomes dark because both of the $\{111\}$ systematic reflections are weakly excited in this area. A new bright band, however, begins to appear at about 1 MV at the symmetry position, ${ }^{22), 25)}$ and its brightness increases markedly as the accelerating voltage increases to $3 \mathrm{MV}$, as seen in Fig. 3b. At the same time, diffraction spots increase in number in 


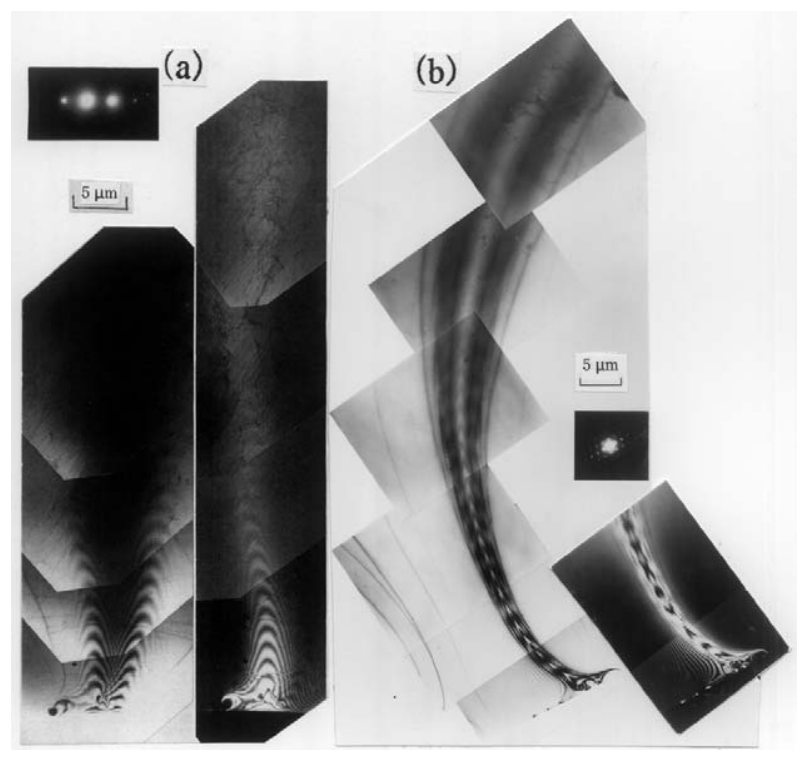

Fig. 3. Voltage dependence of the anomalous transmission of electrons at the symmetry position of the bend contours of the $\{111\}$ reflections in wedge-shaped aluminum specimens. Micrographs $\mathrm{a}$ and $\mathrm{b}$ were taken at 0.5 and $3 \mathrm{MV}$, respectively; each of them shows a bright-field image (left) and a dark-field image (right). Note that a bright band newly appears at the symmetry position in the bright-field image in b where the image is dark in a.

the selected area electron diffraction pattern at the symmetry position, as seen in the inserted diffraction patterns. This means that the degree of simultaneous reflection markedly increases with increasing accelerating voltage, as suggested by Howie. ${ }^{26)}$

The effect of the objective aperture angle on both the value of $\boldsymbol{t}_{M}$ and the image resolution. Most electron microscopists around 1950-70 thought that, in order to acquire electron microscope images of uniform quality, the objective aperture angle must be decreased with increasing accelerating voltage, because the diffraction angle decreases with increasing accelerating voltage if it is assumed that there is only one diffracted wave. This is one reason why they did not pay attention to the effect of simultaneous reflection on the voltage dependence of $t_{M}{ }^{6), 12)}$

Practically, however, the total aberration of the image $\left(\delta_{t}\right)$ consists of the diffraction $\left(\delta_{\text {diff }}\right)$, spherical $\left(\delta_{s p h}\right)$ and chromatic $\left(\delta_{c h r}\right)$ aberrations, as follows:

$$
\delta_{t}=\left(\delta_{\text {diff }}^{2}+\delta_{\text {sph }}^{2}+\delta_{c h r}{ }^{2}\right)^{1 / 2},
$$

where $\delta_{d i f f} \simeq 0.6 \lambda / \alpha, \delta_{s p h}=C_{s} \alpha^{3}$ and $\delta_{c h r}=C_{c} \alpha \Delta E / E$, and

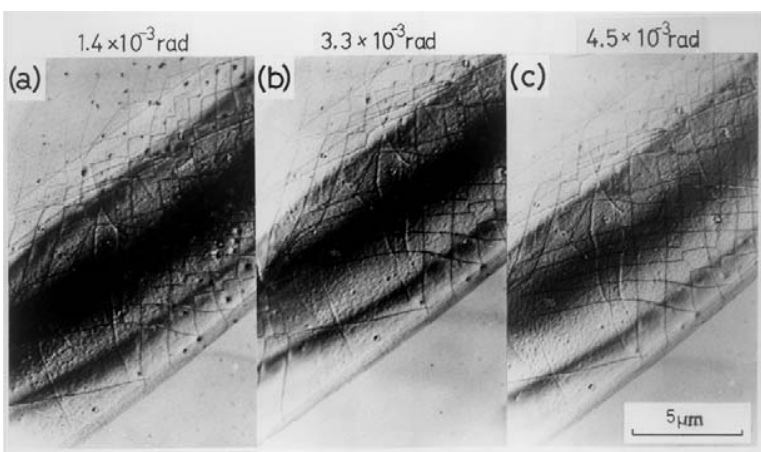

Fig. 4. The effect of the objective aperture angle on the maximum observable specimen thickness at $2 \mathrm{MV}$ for the dislocation images of aluminum.

$\alpha, C_{s}, C_{c}, E$ and $\Delta E$ are the objective aperture angle, the spherical and chromatic aberration coefficients, the energy of electrons and the energy fluctuation, including the energy loss of electrons in materials, respectively. Notably, in Eq. [1] the $\delta_{\text {diff }}$ is divided by $\alpha$, but the other two terms, $\delta_{s p h}$ and $\delta_{c h r}$, are multiplied by $\alpha^{3}$ and $\alpha$, respectively, so that there is an optimum value of $\alpha$ minimizing $\delta_{t}$.

Figures $4 a, b$ and $c$ show the effect of the objective aperture angle $(\alpha)$ on the dislocation images at $2 \mathrm{MV}$, in which not only the brightness of the micrograph but also the sharpness of the dislocation images increases with increasing $\alpha$, indicating in the upper portion. ${ }^{8,27)}$ The diffraction contrast of these images results from the diffracted waves outside of the used aperture, where many diffracted waves are excited at high accelerating voltages. Here, in order to determine the voltage dependence of $t_{M}$, the value of $\delta_{t}$ should be fixed. Figure 5 shows the effect of $\alpha$ on the value of $t_{M} ; t_{0.1}$ is the value of $t_{M}$ at $100 \mathrm{kV}(0.1 \mathrm{MV})$; the ordinate shows the ratio of $t_{M}$ at each voltage against that at $0.1 \mathrm{MV}$. The shaded band in the figure corresponds to the experimental results demonstrating the voltage dependence of the dislocation images for various metals, when the value of $\delta_{t}$ is fixed at $5 \AA^{21)}$ If the value of $t_{M}$ is simply assumed to be proportional to the value of $P$, as they hypothesized, the voltage dependence of $t_{M}$ can be illustrated by the lowest curve of $\alpha=0$ in Fig. $5 .{ }^{28)}$

The shaded curve in Fig. 5 resulted from the increase in the degree of simultaneous reflection in the specimens as the accelerating voltage increased, because strong mutual interaction occurs among these many excited waves at high voltages. This phenomenon 


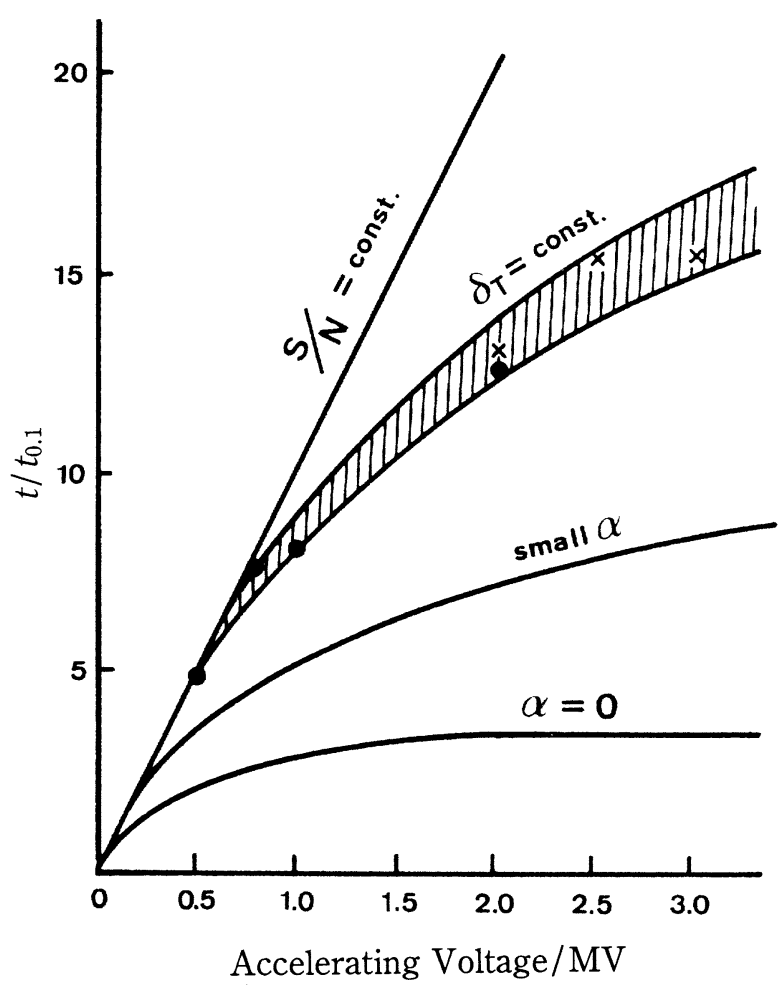

Fig. 5. The effect of the objective aperture angle $(\alpha)$ on the ratio of the maximum observable specimen thickness at each voltage against that at $0.1 \mathrm{MV}$; the shaded band shows the experimental results when the total aberration was fixed at $5 \AA$.

was named "Bloch-wave channeling" in $1971^{29)}$ and also independently named "electron channeling" in $1972 .{ }^{30)} \mathrm{In}$ order to use the simultaneous reflection to enhance the image resolution, the objective aperture angle must cover the corresponding diffraction spots, depending on whether a fixed resolution or the highest resolution is aimed at. According to the classical model proposed by Fujimoto, et al. ${ }^{30)}$ the degree of electron channeling can be expressed by the axial channeling parameter $\left(A_{a}\right)$ along the crystal axis and the planar channeling parameter $\left(A_{p}\right)$ along the crystal plane, as follows:

$$
\begin{aligned}
& A_{a}=4 m Z_{1} Z_{2} e^{2} / h^{2} N d^{2}, \\
& A_{p}=4 m V_{h} / h^{2} b_{h}{ }^{2},
\end{aligned}
$$

where $Z_{1}=1$, and $m, Z_{2}, e, h, N$ and $d$ in Eq. [2] are the relativistic mass of an electron, the atomic number of the crystal atom, the charge of an electron, the Planck constant, the atomic density and the interatomic distance

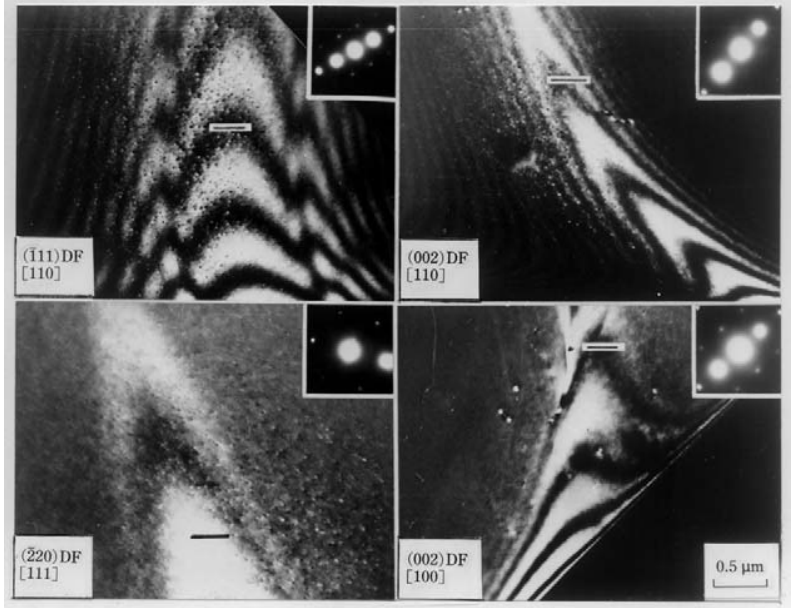

Fig. 6. The orientation dependence of the electron irradiation damage in wedge-shaped aluminum specimens. All micrographs are dark-field images taken at $2 \mathrm{MV}$ and about $300 \mathrm{~K}$.

along the crystal axis, respectively. Furthermore, $V_{h}$ and $b_{h}$ in Eq. [3] are the Fourier coefficient of the crystal potential and the reciprocal vector, respectively. The experimental results show that the classical model is adequate when these two parameters, especially $A_{p}$, are larger than unity, e.g., $A_{p}>1.1{ }^{22), 30)}$

Eqs. [2] and [3] emphasize the fact that both $A_{a}$ and $A_{p}$ are proportional to the relativistic mass of an electron $(m)$, which increases linearly with increasing accelerating voltage, as shown in Fig. 2. Since the value of $t_{M}$ is considered to be proportional to both the value of $A_{a}$ and that of $A_{p}$, especially $A_{p}$, it also increases linearly with increasing accelerating voltage when the corresponding $A_{p}$ is larger than unity, as shown by the curve of the constant signal-to-noise ratio $(S / N)$ in Fig. $5^{8), 19)}$ Practically, however, the voltage dependence of $t_{M}$ must be examined under the condition of fixed image resolution, as shown by the shaded curve in Fig. 5. Remarkably, in Fig. 5 the value of $t_{M}$ increases linearly at least up to about $0.5 \mathrm{MV}$ even along the shaded curve. The value of $t_{M}$ at $3 \mathrm{MV}$ is more than 15 times that at 0.1 $\mathrm{MV}^{21)}$ This value at $3 \mathrm{MV}$ corresponds to $3 \mu \mathrm{m}$ or more in specimen thickness, even for high-Z materials such as tungsten and gold, and to more than $30 \mu \mathrm{m}$ in thickness for aluminum. ${ }^{21)}$ Therefore, at $3 \mathrm{MV}$ the specimen thickness is sufficient to enable the observation of the same phenomena as those in almost all bulk specimens, even for high-Z materials.

Image broadening due to the inelastic scat- 


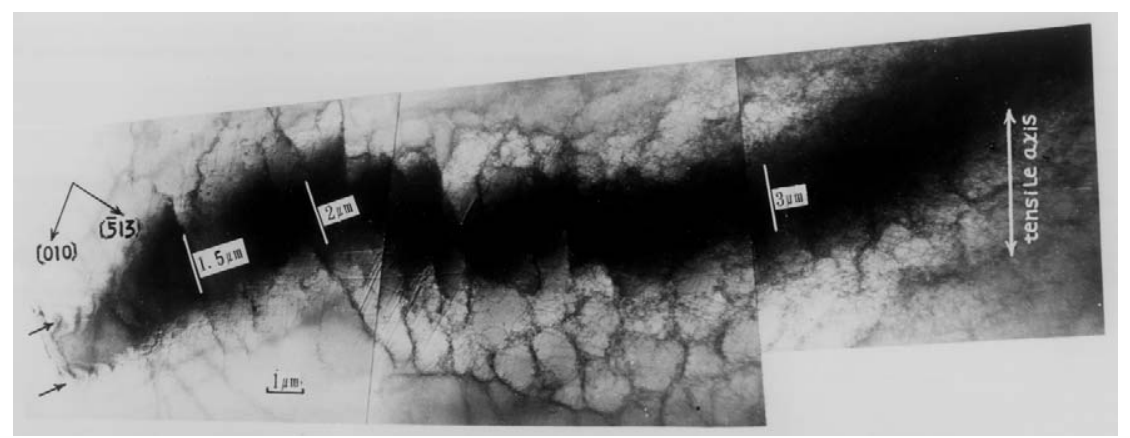

Fig. 7. The effect of thickness on the formation of cell structures in a wedge-shaped aluminum specimen deformed in-situ at $0.5 \mathrm{MV}$ and about 300K.

tering of electrons in materials. One of the aforementioned dire hypotheses was that the images would broaden for thick materials. ${ }^{14)}$ Practically, however, the effect is not so serious, as discussed in the next section, because the image contrast results from the mutual interference of many waves, each of which suffers almost the same energy loss due to the inelastic scattering. For example, the image broadening of lattice defects is scarcely observed even in such light metals as aluminum, at least up to about $15 \mu \mathrm{m}$ in specimen thickness at about $2 \mathrm{MV}^{25)}$

Orientation dependence of electron irradiation damage in materials. Another dire hypothesis was that electron irradiation damage would occur during observation with HVEMs. ${ }^{15)}$ Electron irradiation damage depends on both the energy and the total dosage of electrons within the irradiated area. According to Eqs. [2] and [3], the values of $A_{p}$ and $A_{a}$ are inversely proportional to the square of the reciprocal vector $\left(b_{h}\right)$ and of the interatomic distance $(d)$, respectively. Thus, the intensity of the penetrating electrons decreases markedly when there is an increase in either or both $b_{h}$ and $d .^{25)}$

Figure 6 shows by way of an example this orientation dependence of electron irradiation damage at $2 \mathrm{MV}$ and room temperature in wedge-shaped aluminum specimens. ${ }^{31,32)}$ Each electron micrograph in Fig. 6 is a dark-field image taken under the corresponding individual conditions shown in the parentheses, and electron irradiation was done with the same total dosage of electrons, $4 \times 10^{21} \mathrm{e} / \mathrm{cm}^{2}$. Electron irradiation damage, each of which was measured at the same thickness indicated by a short bar, was marked when electron irradiation was carried out along both the close-packed crystallographic direction, $<110>$, and the plane orientation, $\{111\}$, as seen in the left upper micrograph. The right upper micrograph was taken after the order of reflection was changed from $\{111\}$ to $\{002\}$ and under the same incident direction of electrons; the degree of irradiation damage, i.e., the total volume of secondary defects, decreased. The electron irradiation damage was further reduced when the incident direction of electrons was changed from $<110>$ to $<100>$ under a fixed reflection of $\{002\}$, as seen in the lower right micrograph.

Generally, a TV-VTR system is used for image recording with $\mathrm{HVEMs}^{33)}$ and ultra-HVEMs, ${ }^{8), 19)}$ and this further decreases the electron irradiation damage, by more than three orders of magnitude. Practically, serious problems can be suppressed under the aforementioned suitable conditions even after electron microscope observation for more than $1.8 \mathrm{ks}$ at accelerating voltages higher than $2 \mathrm{MV}$.

In contrast, high $A_{p}$ and $A_{a}$ values can be effectively used in the investigation of electron irradiation damage and related phenomena in materials. ${ }^{25), 32)}$

The effect of thickness on various phenomena. As stated already, even in cold-worked specimens, no dislocation is observed in regions whose thickness is less than the critical thickness, which is a function of the dislocation density and the kind of material involved; e.g., the critical thickness of aluminum is about $0.2 \mu \mathrm{m}$ at $10^{10} / \mathrm{cm}^{2}$ in dislocation density, but increases to $0.8 \mu \mathrm{m}$ when the dislocation density decreases to $10^{6} / \mathrm{cm}^{2}$. Figure 7 shows the effect of thickness on the formation of dislocation cell structures through in-situ deformation of a wedge-shaped aluminum specimen. In that specimen, the local thicknesses are precisely determined by the combination of equal-thickness fringes and twodimensional structures, such as slip planes, whose traces were formed after in-situ observation. ${ }^{7,25)}$ Notably, the same dislocation cell structures as those in 
Table I. The accelerating voltages necessary for the study of various research subjects

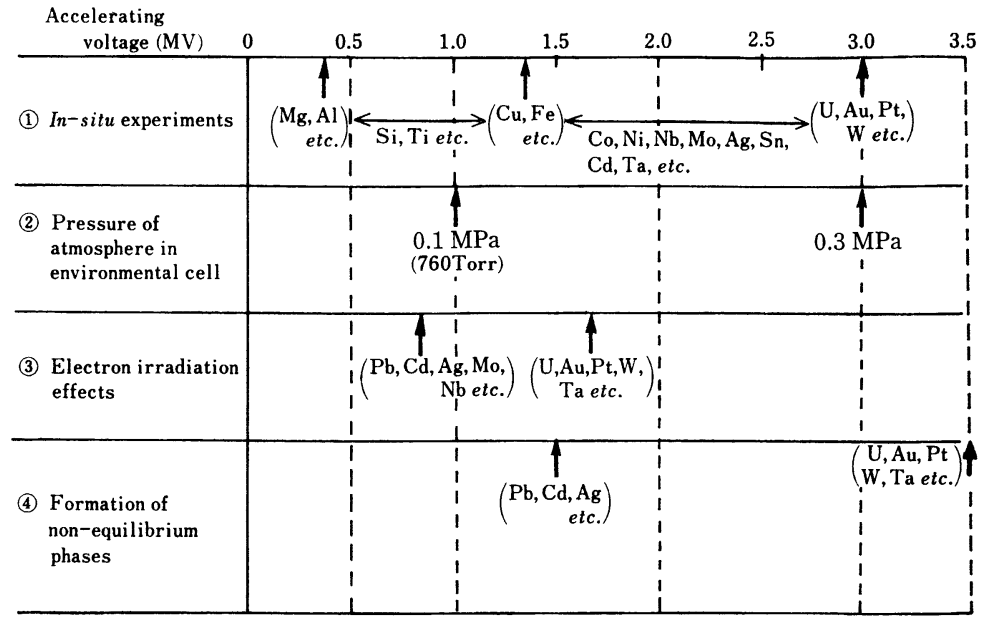

bulk specimens are formed in regions whose thickness is larger than about $3 \mu \mathrm{m},{ }^{5)}$ as shown in Fig. 7. Furthermore, recrystallization and successive grain growth occur in both aluminum and Fe-Si alloys where the thickness of the specimen is larger than about $1 \mu \mathrm{m},{ }^{4,7)}$ but only subgrains are formed in thinner regions.

The aforementioned results show that, in order to obtain the same phenomena as in bulk specimens, the specimen thickness must always be larger than the mean free path of the lattice defects contributing to the phenomena, e.g., about $3 \mu \mathrm{m}$ or more for dislocations in general. The value of $t_{M}$ increases beyond $3 \mu \mathrm{m}$ at $3 \mathrm{MV}$ for most materials, as shown in Fig. 5, and thus in-situ observation of various phenomena in bulk specimens made of those materials can be carried out at $3 \mathrm{MV}$. Based on results corresponding to those for bulk specimens, the effect of size on various phenomena, including the anomalous change in behavior of nanometer-sized specimens, can be investigated with precision.

In-situ observation with ultra-HVEMs. Since the phenomena representative of most bulk materials can be investigated dynamically with HVEMs ${ }^{4)-7), 33)}$ and ultra-HVEMs, ${ }^{8), 19), 25)}$ various specimen treatment devices have been improved ${ }^{8)}$ so that a 3 MV ultra-HVEM can carry out in-situ observation. Specimen manipulation, such as stretching, alternating stressing, constant-load deformation, electron irradiation under various conditions, treatment in various environments with pressures up to $0.3 \mathrm{MPa}$, and combinations of these can be carried out in a wide temperature range, from about 10 to $2,300 \mathrm{~K}$. All of these devices are of the top-entry type and are mounted on a universal goniometer stage whose maximum tilting angle is more than $10^{\circ}$. Table I shows the accelerating voltages necessary for various applications in materials science. ${ }^{8}$ Note that the accelerating voltage must be selected depending on the purpose, ${ }^{4)-7), 8), 33)}$ and that 3 MV-class ultra-HVEMs cover the needs of almost all research subjects, even when very high-Z materials are examined. ${ }^{8)}$

The mechanisms behind the following phenomena have been made clear by in-situ observation: (1) the dislocation behavior during both yielding and work-hardening under tensile stress, (2) the effects of crystal orientation, stacking fault energy and deformation temperature on the dislocation behavior, (3) the dislocation behavior during fatigue and creep deformation, and in the vicinity of grain boundaries and crack tips, (4) the dislocation behavior in bcc crystals both under high-energy electron irradiation and in a hydrogen atmosphere, (5) the role of dislocations in the melting process, (6) the formation of mechanical twins, (7) the martensitic transformation from an fcc structure to an hcp or a bcc structure, (8) the recovery and recrystallization of metals and alloys, (9) the formation of G.P. zones and other phases in Al-Cu alloys, (10) the formation of primary and secondary defects induced by electron irradiation under various conditions, (11) the temperature dependence of critical voltage phenomena, (12) sintering by epitaxial recrystallization, (13) the deformation of highly toughened $\mathrm{ZrO}_{2}-\mathrm{Y}_{2} \mathrm{O}_{2}$ composites and the superelasticity of $\mathrm{ZrO}_{2}-\mathrm{MgO}$ composites, (14) the 

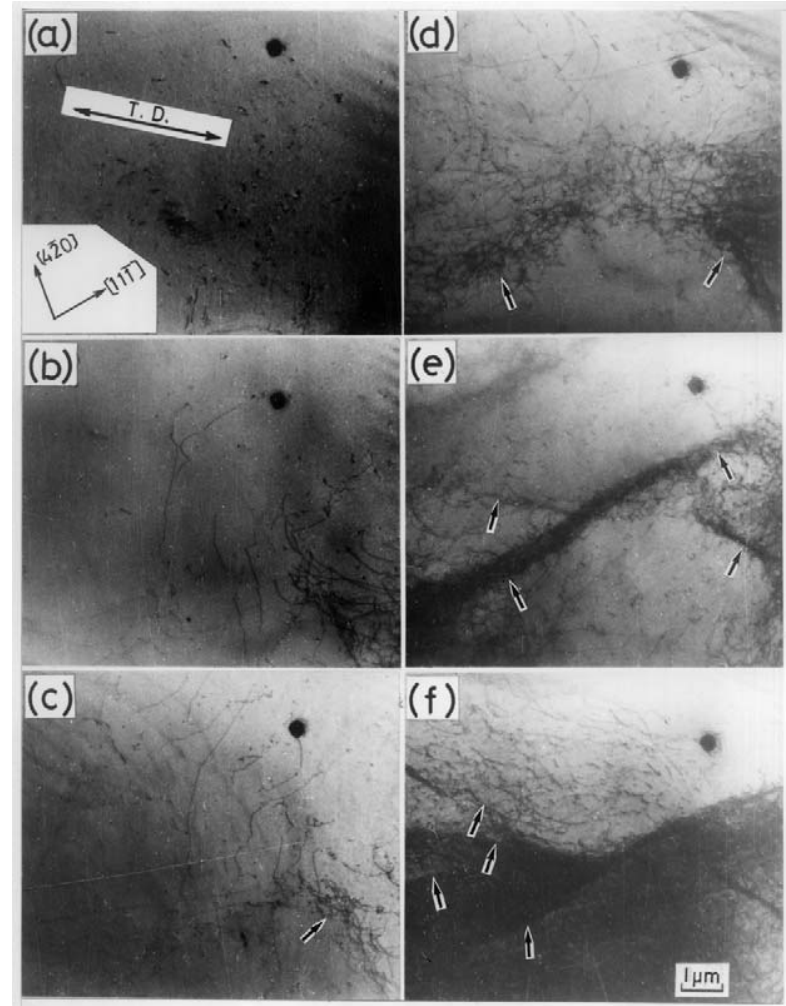

Fig. 8. The movement of dislocation tangles $(\uparrow)$ during the deformation of aluminum. The micrographs were taken at 0.5 MV and about $300 \mathrm{~K}$, and each black spot in the upper portion shows a fixed position.

sintering of $\mathrm{Al}_{2} \mathrm{O}_{3}-\mathrm{ZrO}_{2}$ composites and the elimination of pores by migrating boundaries in ceramic composites, etc.

In the acquisition of these results, in-situ observation plays an important role in elucidating the dislocation behavior, especially on the following points: (a) the movement of each individual dislocation, which is easily distinguished due to its slip trace formed for an instant by the interaction between the specimen surface and each moving dislocation, and (b) the shape and distribution of the dislocation lines, which are widely different when the applied stress is released, i.e., under static observation.

Furthermore, closed-type environmental cells have been applied to the following activities ${ }^{8)}$ : (15) the chemical amorphization of $\mathrm{Zr}$-Al alloys through the absorption of $\mathrm{H}_{2}$ and $\mathrm{O}_{2}$ gases, (16) the inner oxidation of metals and alloys, (17) the observation of wet materials, including biological ones, etc.

Based on the aforementioned experimental results, the mechanisms behind various phenomena
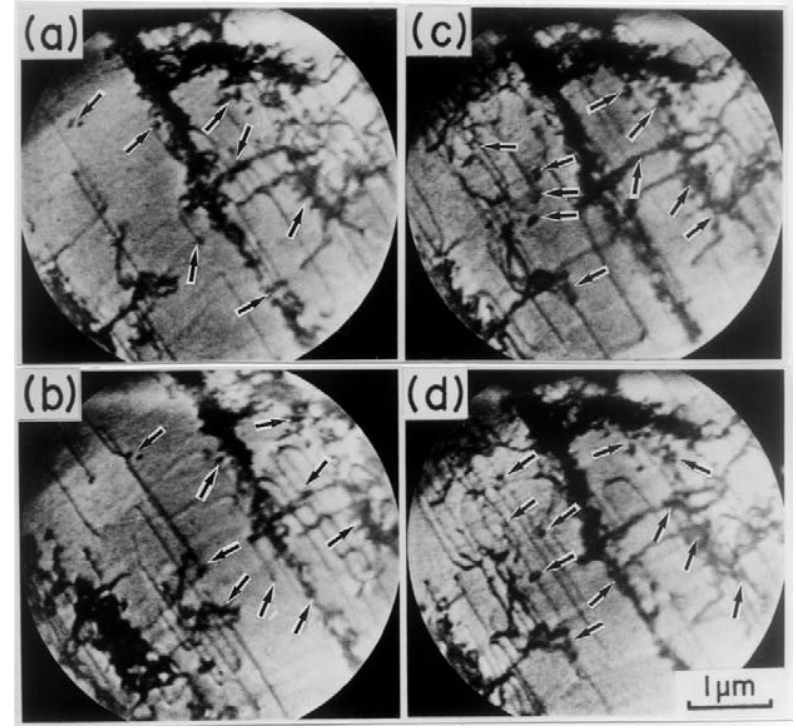

Fig. 9. The process of formation of dislocation tangles in the $<111>$ W single crystal at a low homologous temperature, i.e., $0.09 \mathrm{Tm}$ (Tm: the absolute melting temperature). The micrographs were picked out from a TV-VTR tape taken at 2 $\mathrm{MV}$ and about $300 \mathrm{~K}$. The arrows show prismatic dislocation loops.

have been clarified. ${ }^{8), 31,34)}$ There are some results, which constitute new evidence in support of the current conclusions, as follows: First, the behavior of the dislocation source: a dislocation source generally makes a cross-slip at the beginning, and then the dislocation multiplies ${ }^{35)}$; after that, the multiplied dislocations intersect with other dislocations of different slip systems during their movement, so that they always make a number of jogs even when the stacking fault energy is very low ${ }^{36)}$; furthermore, a dislocation source generally multiplies only into several dislocations in general, and then decomposes by moving out its pole dislocations, ${ }^{35)}$ due to the fact that the mobility of the dislocations depends not only on Schmid's factor for the slip planes but also on their length. Second, the interaction between multi-slip systems: at least two slip systems are generally activated regardless of the stacking fault energy, and dislocation cell structures and/or hexagonal networks of dislocations are formed by the interaction of those slip systems during deformation, depending on the stacking fault energy, which is very low in hexagonal networks. ${ }^{37)}$ These dislocation networks ${ }^{37)}$ and dislocation cell structures ${ }^{38,39)}$ are generally mobile during deformation, as shown in the images of Figs. 8 and 9 taken from aluminum and tung- 


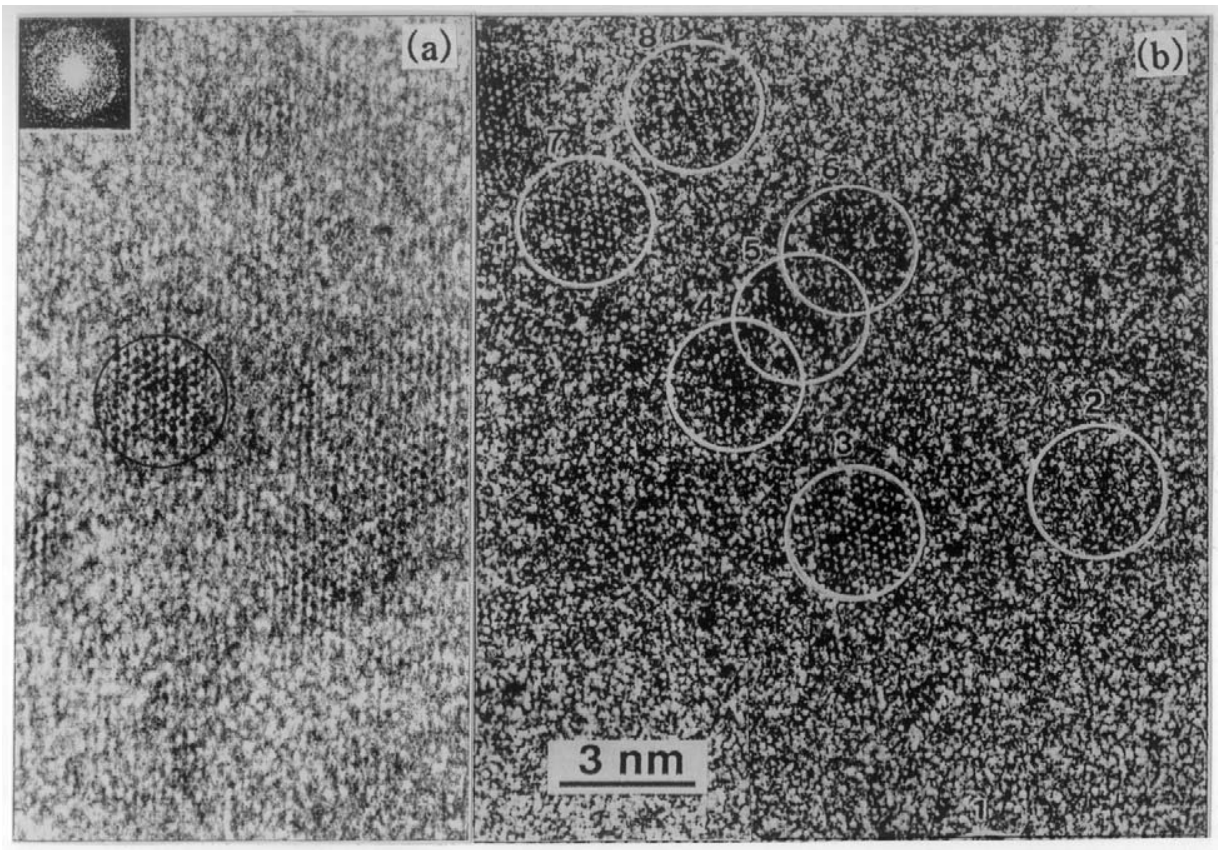

Fig. 10. The critical size reflecting the periodic structure of the constituent atoms in a Ti-50at\%Ni alloy. Micrograph a shows an intermediate stage of the crystalline-to-amorphous transition induced by electron irradiation at $2 \mathrm{MV}$ and $170 \mathrm{~K}$, and b shows the crystal nucleation of the fully amorphized alloy induced by in-situ annealing at $573 \mathrm{~K}$ for $1.2 \mathrm{ks}$. In both micrographs, the encircled regions show the critical size, respectively.

sten specimens, respectively, whereas to date they have been considered sessile during deformation. Remarkably, Figs. 8 and 9 show that the tangling of dislocations generally occurs around the prismatic dislocation loops formed by double cross-slips, after which dislocation cell structures are formed. ${ }^{38), 39)}$ These dislocation cell structures in Fig. 8 move to regions (the upper portion of the micrograph) where the moving dislocations arrive. ${ }^{38)}$ Furthermore, the individual dislocations of the cell structures in Fig. 9 move out in succession every time a new dislocation moves into the cell structures. ${ }^{39)}$ Third, the boundary energy of recrystallized grain is a function of the dislocation density within the recrystallized region, consisting of a subgrain group, during recrystallization, ${ }^{17), 40)}$ whose process is also called "subgrain coalescence". ${ }^{17)}$ Fourth, grains and particles of $\phi 0.1 \mu \mathrm{m}$ or less in size play an important role in the strength ${ }^{41), 42)}$ and the sintering of materials, ${ }^{43}$ ) respectively.

Various quantitative measurements can also be carried out for thick materials, as follows: (1) since insitu observation can be carried out to cover a wide region, the results for the interior structures are easily correlated with the images of macroscopic surface structures obtained with optical microscopes, ${ }^{37)}$ and (2) various quantitative measurements, such as threedimensional data on microstructures, and the determination of such physical parameters as the migration energy of point defects, etc., can be carried out with precision. $^{36)}$

Indispensable applications of ultra-HVEMs. Besides the aforementioned in-situ observation of various phenomena, there are other indispensable applications of ultra-HVEMs, such as foreign atom implantation and the formation of non-equilibrium phases.

Crystalline-to-amorphous transition of mate-

rials. To date, amorphization has been induced by highenergy electron irradiation at low temperatures in 60 intermetallic compounds and 30 ceramics. ${ }^{44)}$ Based on the experimental results, the general rules of amorphization have been formulated. ${ }^{36)}$ The most important result is the discovery of a critical size for amorphization. Figures $10 \mathrm{a}$ and $\mathrm{b}$ were picked out from successive micrographs showing the crystalline-to-amorphous 
transition and the reverse process in a Ti-50at\% $\mathrm{Ni}$ alloy, respectively. The specimen is a typical shape-memory alloy, and is difficult to amorphize even by the liquid quenching method. Amorphization of this alloy had been carried out by electron irradiation at $2 \mathrm{MV}$ and 170 K, and Fig. 10a shows an intermediate stage of the amorphization. ${ }^{45)}$ Notably, the micrograph shows that the periodic structure of the constituent atoms disappears when the size of the region becomes smaller than the critical one. Namely, there is a minimum size, $\phi 3 \mathrm{~nm}$ for this alloy, above which multi-wave lattice fringes are observed, as encircled in the micrograph. This means that there is a critical size for maintaining the crystalline state, under which the periodic structure is disrupted. Since the crystallization of amorphous materials is the reverse of amorphization, the Ti-50at\% Ni alloy fully amorphized by electron irradiation was annealed at 573 $\mathrm{K}$ for $1.2 \mathrm{ks}$, as shown in Fig. 10b. ${ }^{46)}$ It is noteworthy that the multi-wave lattice fringes in Fig. 10b appear in limited regions, each of which always has the same size, $\phi 3$ $\mathrm{nm}$, at the beginning of crystallization. This means that the size of the limited regions corresponds to the critical size for the crystalline state, i.e., the size of the crystal nucleus. Here, the temperature difference between the amorphization shown in Fig.10a and the crystal nucleation shown in Fig. 10b is quite large, i.e., larger than 400 $\mathrm{K}$. This means that the critical size necessary for the onset of the crystalline state in the Ti-50at\% $\mathrm{Ni}$ alloy is always $\phi 3 \mathrm{~nm}$ in such a wide temperature range.

Furthermore, inducing the transition from the amorphous to the crystalline state is the most effective method of determining the nucleus size of crystals, because the incubation time for the growth of the nuclei becomes considerably large, so that the nuclei always have the same size in a wide temperature range. ${ }^{46)}$ Since the electronic structure of a material is a function of its atomistic structure, the determination of the nucleus size of crystals is very important for establishing the critical size, under which the behavior of the material changes anomalously, as discussed later.

Foreign atom implantation into substrate materials through high-energy electron irradiation. Using high-energy electron irradiation at sufficiently low temperatures, foreign atoms can be implanted into substrate materials. Figure 11 shows $\mathrm{Pb}$ atoms implanted into an aluminum substrate. ${ }^{47)}$ In this method, the substrate must be kept at low temperatures (e.g., $170 \mathrm{~K}$ ), so that the excess point defects induced by the electron irradiation do not migrate. The image contrast of the implanted regions at room temperature does not

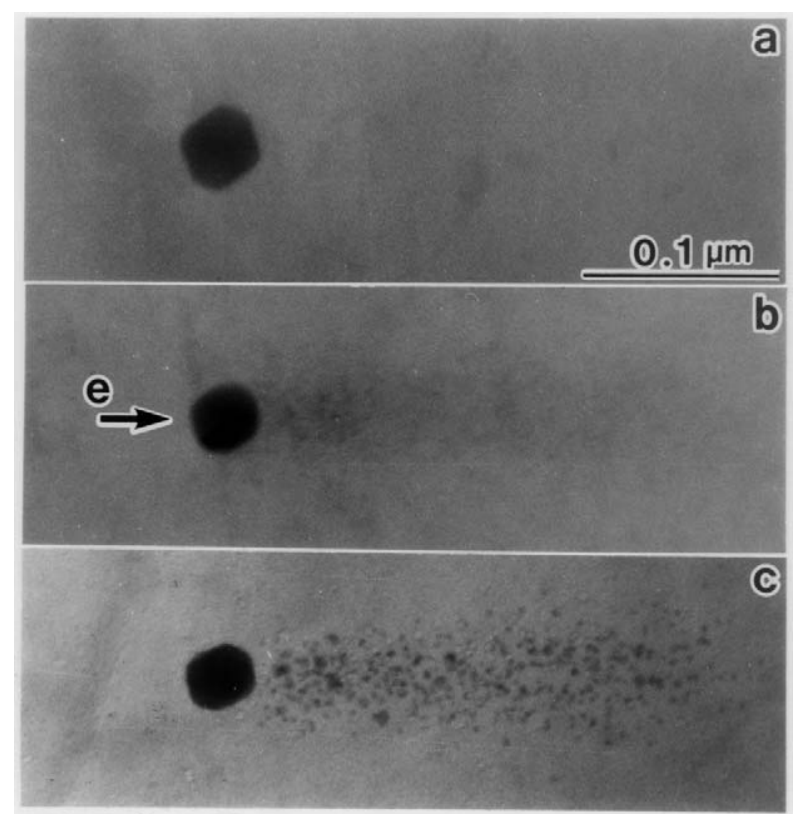

Fig. 11. Micrographs a and $\mathrm{b}$ were taken before and after the implantation of $\mathrm{Pb}$ atoms into an aluminum substrate at $2 \mathrm{MV}$ and $170 \mathrm{~K}$, and $\mathrm{c}$ was taken after post-annealing at $575 \mathrm{~K}$ for 36 ks. Both a large block and ultrafine particles in the micrographs represent $\mathrm{Pb}$ particles. Electron irradiation was carried out at a dosage of $1.08 \times 10^{28} \mathrm{e} / \mathrm{cm}^{2}$ along the direction indicated by the arrow, at a rate of $1.2 \times 10^{24} \mathrm{e} / \mathrm{cm}^{2} / \mathrm{s}$.

increase much despite the large difference (more than $22 \%$ ) in atomic size between the two elements, as shown in Fig. 11b. After annealing at $575 \mathrm{~K}$ for $36 \mathrm{ks}$, the implanted $\mathrm{Pb}$ atoms precipitated as ultrafine particles (Fig. 11c), and many voids appeared simultaneously in the implanted region. The results show that the implanted atoms interact successively with these excess point defects, so that the lattice distortion around each implanted atom is reduced. ${ }^{36)}$ As a result, the implantation of oversized atoms is enhanced by the interaction with the excess vacancies, whereas the implantation of undersized atoms is enhanced by the interaction with the excess interstitials.

Foreign atom implantation by this method is superior to that achieved by other methods such as ion implantation, as follows: (a) there is only a small lattice distortion due to the relaxation induced by the point defects, (b) the specimen shows only a small rise in temperature, i.e., a few degrees, during implantation, (c) the foreign atoms are implanted deeply, e.g., at a depth of up to $10 \mu \mathrm{m}$ or more at $2 \sim 3 \mathrm{MV}$, depending on the kinds of materials involved, (d) foreign atoms can be implanted 
into extremely fine portions of the material with a fine electron beam whose cross-section is minimized to about $\phi 1 \mathrm{~nm}$, and thus a complex electronic circuit made of different elements can be directly impressed into the substrate with the scanning mode of the ultrafine electron beam, (e) foreign atom implantation can be carried out at any location within the substrate material, as long as the electron energy is sufficiently high, and (f) provided that the conditions of foreign atom implantation are adequate, such as the existence of a large difference in the collision cross-section between guest and host atoms, a particle of guest atoms (e.g., a gold particle) can be implanted as a block into the host material (e.g., silicon crystal). ${ }^{48)}$

Figure11 is also direct proof of electron channeling, i.e., the passage of high-energy electrons along the incident direction.

Table II. The value of $d_{M}$ for various materials, as a function of the bonding mode, the binding strength and the surrounding conditions.

\begin{tabular}{ccll}
\hline \multicolumn{2}{c}{ Magic size $(\phi \mathrm{nm})$} & \multirow{2}{*}{ Bonding mode of materials } \\
\cline { 1 - 2 } Isolated & Embedded & \\
\hline $20-8$ & $8-4$ & & Metallic and ionic bonding \\
$6-3$ & $4-2$ & & Intermetallic compounds \\
$3-1.5$ & $2-1$ & & Covalent and diamond bonding \\
\hline
\end{tabular}

In-situ observation of the anomalous behavior of atom clusters. As stated already, the behavior of materials changes anomalously when the size of the material becomes smaller than the critical size, on the nanometer scale. The critical size $\left(d_{M}\right)$, which is also called "the magic size", and the characteristics of atom clusters (ACs) have been successfully investigated using ultra-HVEMs. ${ }^{49)-51)}$

The anomalous behavior of ACs cannot be understood on the current simple assumption that the behavior of materials is markedly changed by the surface, when the number of atoms constituting the surface becomes larger than that constituting the interior, i.e., when the total number of constituent atoms becomes smaller than about $3 \times 10^{2}$, regardless of the kind of material. The experimental results, however, show that the critical number of constituent atoms widely varies from about $10^{3}$ to $10^{5}$ atoms; this number range corresponds to $\phi 1 \mathrm{~nm}$ to $\phi 20 \mathrm{~nm}$ in size, depending on both the kind of elements involved and the surrounding conditions, as shown in Table II. ${ }^{49), 51)}$

The spontaneous mixing of guest atoms, including gas atoms, into the ACs of the host material is a typical example of anomalous AC behavior, and it occurs spontaneously even at temperatures lower than $300 \mathrm{~K}^{52), 53)}$ Figure 12 shows an example of the effect of size on the spontaneous mixing of guest $\mathrm{Cu}$ atoms into host $\mathrm{Au}-\mathrm{ACs}$ at $300 \mathrm{~K}$. The average size of the original Au particles is
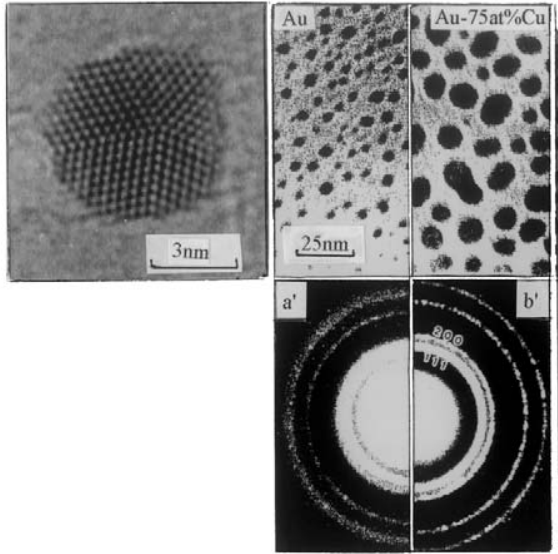

(a)

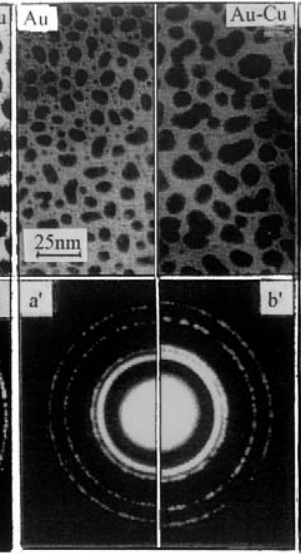

(b)

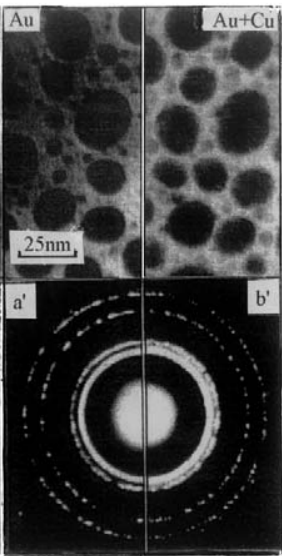

(C)

Fig. 12. The effect of size on the spontaneous mixing of $\mathrm{Cu}$ atoms into ultrafine particles of $\mathrm{Au}$ which were deposited onto an amorphous carbon film. The right and left micrographs of each set show images before and after the in-situ vapor deposition of the $\mathrm{Cu}$ atoms onto the Au particles, and the marks a' and b' are the corresponding diffraction patterns, respectively. A highresolution micrograph of the Au particles is shown at the upper left corner. 
$\phi 5,10$ and $20 \mathrm{~nm}$ in micrographs a, b and c, respective$\mathrm{ly}$. When the $\mathrm{Cu}$ atoms were vapor-deposited onto the $\mathrm{Au}$ particles in a vacuum (micrograph a), the former were rapidly absorbed into the latter, as indicated by the rapid expansion of the diffraction rings, to form a solid solution of $\mathrm{Au}-75 \mathrm{at} \% \mathrm{Cu}$ alloy. Micrograph b shows the same phenomenon, but micrograph $\mathrm{c}$ shows that the $\mathrm{Cu}$ atoms only covered the surface of the $\mathrm{Au}$ particles when the size of the Au particles increased to $\phi 20 \mathrm{~nm}$, as indicated by the diffraction pattern, in which the diffraction rings of both $\mathrm{Au}$ and $\mathrm{Cu}$ were superimposed. The result shows that spontaneous mixing occurs when the particle size is smaller than the $d_{M}$ of isolated Au-ACs, namely at about $\phi 15 \mathrm{~nm}$. The spontaneous mixing of guest atoms into host-ACs can be effectively exploited not only in making various alloys even when the specific gravity of the host and guest atoms is widely different, but also in making fuel batteries through the anomalous absorption of such gas atoms as $\mathrm{H}$ and D into host-ACs. ${ }^{54)}$

Furthermore, the ordered structure, e.g., the $\mathrm{Au}-\mathrm{Cu}$ alloy, ${ }^{55)}$ decomposes into a solid solution when the size of the alloy becomes smaller than about $\phi 4 \mathrm{~nm}$. This is also due to the spontaneous mixing of the guest atoms into the ACs of the host material. ${ }^{51,55)}$

Here, the value of $d_{M}$ is exactly determined by the nucleus size during crystal nucleation, ${ }^{46)}$ and also from the anomalous change in behavior of the materials. Also, the value of $d_{M}$ (Table II) decreases not only with increasing bonding covalency, but also with decreasing coordination number. The value of $d_{M}$ for two- and onedimensional ACs can also be roughly estimated from the effect of the shape on the atomistic structure of the material. ${ }^{49)}$

Conclusions. Electron channeling is strongly excited by simultaneous reflections at high voltages, and leads to a remarkable increase of the maximum observable specimen thickness, by using the effect of the objective aperture angle, with no loss of visibility in the electron microscope images. The result has been the development of epoch-making applications in materials science with the help of ultra-HVEMs, as follows: (a) the in-situ high-precision observation of phenomena representative of most bulk materials under various conditions, including environmental ones, at least on the scale of lattice defects even in high-Z materials, and the clarification of the mechanisms behind those phenomena (these results are also very important to the investigation of the effect of size on the behavior of nanometer-sized materials); (b) the effective suppression of electron irradiation damage through the selection of a suitable crystal orientation; (c) the in-situ observation of wide regions, so that the obtained results are easily correlated with the surface structures observed with optical microscopes; (d) the high-precision quantitative measurement of various features of the material, such as the three-dimensional shape of microstructures, and the determination of physical parameters, etc.

Indispensable applications of ultra-HVEMs have also been carried out, as follows: (1) the implantation of foreign atoms directly into substrate materials through high-energy electron irradiation; (2) the high-precision study of the crystalline-to-amorphous transition in various materials, which resulted in the discovery of both the nucleus size of the crystals and the general rules of amorphization; (3) the detailed investigation of the anomalous behavior of atom clusters. The results show that the critical size, under which the behavior of materials changes anomalously, corresponds to the nucleus size of the crystals. New functional materials result from combinations of these items.

Besides the applications mentioned above, the technical options provided by ultra-HVEMs also contribute to the development of various other fields, as seen in the remarkable improvement of the functional features of conventional EMs, which can now clarify atomistic structures $1 \AA$ in spacing, ${ }^{56)}$ in ultrafine fabrication, etc.

Acknowledgements. Most of the experimental results in the present report were obtained with a $3 \mathrm{MV}$ ultra-HVEM at the Research Center for Ultra-high Voltage Electron Microscopy, Osaka University. The author wishes to express his heartfelt thanks to Professor H. Mori and Doctors H. Yamada, T. Tabata, H. Yasuda (currently a Professor at Kobe University), N. Sumida (currently a Professor at Kagawa University), M. Komatsu (currently Associate Professor at Hiroshima Institute of Technology), K. Yoshida and T. Sakata for their help in the experiments at the Research Center. The author also expresses his thanks to Professor K. Ura and all members of the Hitachi ultra-HVEM group for their contribution in constructing the 3 MV ultraHVEM.

Appendix. Since 1965, many HVEMs and a few ultra-HVEMs have been installed around the world, as shown in Table III, in which marks $\bigcirc, \triangle, X$ and ? show good, modelate, workless and uncertain in operating condition at present, respectively. The maximum accelerating voltages of EMs should be shown at least with electron micrographs taken at those voltages, but some of them are shown only with the electron diffraction or 
Table III. A list of HVEMs and ultra-HVEMs which have been installed around the world. This table is based on a report by Hitachi Ltd., and is partly corrected by the author to the best of his knowledge.

\begin{tabular}{|c|c|c|c|c|c|}
\hline Year & Model & $\begin{array}{l}\text { Maximum } \\
\text { voltage(MV) }\end{array}$ & $\begin{array}{l}\text { Set up } \\
\text { place }\end{array}$ & EM-maker & $\begin{array}{c}\text { Present } \\
\text { condition }\end{array}$ \\
\hline 1941 & & 0.3 & USA & RCA & $x$ \\
\hline 1947 & & 0.4 & Netherlands & Philips & $\times$ \\
\hline 1954 & & $0.3^{*}$ & JPN & Hitachi & $x$ \\
\hline 1957 & SMH-3 & 0.3 & JPN & Shimadzu & $\times$ \\
\hline 1958 & & 0.4 & Soviet & Popor & $\times$ \\
\hline 1962 & & $1.2^{*}$ & France & Toulouse & $\times$ \\
\hline 1965 & SMH-5A & 0.5 & JPN(NRIM) & Shimadzu & $\times$ \\
\hline 1965 & HU-500 & 0.5 & JPN & Hitachi & $x$ \\
\hline 1965 & HU-500 & 0.5 & JPN & Hitachi & $\times$ \\
\hline 1966 & SMH-5A & 0.5 & JPN & Shimadzu & $x$ \\
\hline 1966 & SMH-5B & 0.5 & JPN(NRIM) & Shimadzu & $\times$ \\
\hline 1966 & HU-1000 & $1.0 *$ & JPN & Hitachi & $x$ \\
\hline 1966 & JEM-1000 & $1.0 *$ & JPN & JEOL & $\times$ \\
\hline 1966 & & 0.75 & England & Cambridge $^{+}$ & $\times$ \\
\hline 1967 & & 1.0 & USA & $\mathrm{RCA}^{+}$ & $x$ \\
\hline 1967 & & 1.0 & England & AEI & $x$ \\
\hline 1968 & & 1.0 & England & AEI & $\times$ \\
\hline 1968 & SMH-5A & 0.5 & JPN & Shimadzu & $\times$ \\
\hline 1968 & HU-650 & 0.65 & W-Germany & Hitachi & $x$ \\
\hline 1968 & & 0.5 & USA & $\mathrm{RCA}$ & $\times$ \\
\hline 1968 & & 1.0 & Netherlands & Philips & $\times$ \\
\hline 1968 & HU-650 & 0.65 & USA & Hitachi & $\times$ \\
\hline 1969 & HU-650 & 0.65 & JPN & Hitachi & $\times$ \\
\hline 1969 & JEM-1000 & 1.0 & Sweden & JEOL & $\times$ \\
\hline 1970 & HU-650 & 0.65 & USA & Hitachi & $\times$ \\
\hline 1970 & HU-650 & 0.65 & USA & Hitachi & $\times$ \\
\hline 1970 & HU-2000 & 2.0 & JPN(Osaka) & Hitachi & 0 \\
\hline (1972) & HU-2000 & 3.0 & JPN(Osaka) & Hitachi & $x$ \\
\hline 1970 & & 1.0 & England & AEI & $x$ \\
\hline 1970 & & 1.0 & England & AEI & $\times$ \\
\hline 1970 & HU-1000 & 1.0 & England & Hitachi & $x$ \\
\hline 1970 & & 1.0 & England & AEI & $x$ \\
\hline 1971 & & 2.0 & France & Toulouse & $\times$ \\
\hline (1973) & & 2.5 & France & Toulouse & $\times$ \\
\hline 1971 & HU-650 & 0.65 & JPN & Hitachi & $x$ \\
\hline 1971 & JEM-1000 & 1.0 & E-Germany & JEOL & $\times$ \\
\hline 1972 & HU-1000 & 1.0 & JPN & Hitachi & O \\
\hline 1972 & JEM-1000 & 1.0 & USA & JEOL & $?$ \\
\hline 1972 & JEM-1000 & 1.0 & USA & JEOL & $?$ \\
\hline 1972 & JEM-1000 & 1.0 & USA & JEOL & $?$ \\
\hline 1972 & JEM-1000 & 1.0 & JPN & JEOL & $\triangle$ \\
\hline 1972 & & 1.0 & England & AEI & $?$ \\
\hline 1973 & JEMH-500 & 0.5 & JPN & JEOL & $?$ \\
\hline 1973 & & 1.0 & England & AEI & $?$ \\
\hline 1974 & JEM-1250 & 1.25 & JPN & JEOL & ? \\
\hline 1975 & H-1000 & 1.0 & JPN & Hitachi & $x$ \\
\hline 1975 & JEM-1000 & 1.0 & Soviet & JEOL & $?$ \\
\hline 1975 & JEM-1000 & 1.0 & JPN & JEOL & $x$ \\
\hline 1975 & JEM-1250 & 1.25 & JPN & JEOL & $\times$ \\
\hline 1976 & H-1250 & 1.25 & JPN & Hitachi & $x$ \\
\hline 1976 & JEM-1250 & 1.25 & JPN & JEOL & $\bigcirc$ \\
\hline 1977 & JEM-1000 & 1.0 & R-China & JEOL & $?$ \\
\hline 1978 & & 1.0 & USA & AEI & $?$ \\
\hline 1978 & & 1.0 & W-Germany & AEI & $\times$ \\
\hline 1979 & & 1.25 & USA & AEI & $\times$ \\
\hline 1980 & & 1.0 & France & AEI & $?$ \\
\hline 1981 & H-1250S & 1.25 & JPN & Hitachi & 0 \\
\hline 1981 & H-1300 & 1.3 & JPN & Hitachi & \\
\hline 1982 & H-1250M & 1.25 & JPN & Hitachi & \\
\hline 1983 & $\mathrm{H}-1250 \mathrm{ST}$ & 1.25 & JPN & Hitachi & \\
\hline 1983 & JEMA-1000 & 1.0 & USA & JEOL & $\bigcirc$ \\
\hline 1983 & & 1.5 & USA & Kratos/AEI & $?$ \\
\hline 1989 & JEMA-1000 & 1.0 & JPN & JEOL & 0 \\
\hline 1990 & H-1500 & 1.5 & JPN & Hitachi & $\bigcirc$ \\
\hline 1991 & JEMA-1250 & 1.25 & JPN & JEOL & 0 \\
\hline 1992 & JEMA-1250 & 1.25 & JPN & JEOL & 0 \\
\hline 1993 & JEMA-1250 & 1.25 & Germany & JEOL & 0 \\
\hline 1994 & JEMA-1000 & 1.0 & JPN & JEOL & $\bigcirc$ \\
\hline 1995 & H-3000 & 3.5 & JPN(Osaka) & Hitachi & $\bigcirc$ \\
\hline 1998 & JEMA-1300 & 1.3 & JPN & JEOL & 0 \\
\hline 1999 & $\mathrm{H}-1000 \mathrm{~F}$ & 1.0 & JPN & Hitachi & $\bigcirc$ \\
\hline 2002 & JEMA-1300 & 1.3 & Korea & JEOL & $\bigcirc$ \\
\hline
\end{tabular}

those voltages specified in Table III.

Note that the high-voltage generator systems of all HVEMs and ultra-HVEMs in Table III consist of a Cockcroft-Walton circuit and a cascade-type accelerator made of multi-stage electrodes. Some electron microscope specialists in 1960-70 considered that the linear accelerator becomes effective when the accelerating voltage increases to more than $1 \mathrm{MV}$. Actually, however, they could not stabilize the electron energy sufficiently to take electron microscope images with proper resolutions, and thus no practical HVEM and ultra-HVEM using a linear and/or superconducting linear accelerator were available.

On the other hand, many HVEMs have had serious troubles with mechanical vibration proofing. Two $500 \mathrm{kV}$ HVEMs at NRIM succeeded in taking high-resolution images ${ }^{57)}$ by using a double-tank system connected with a high-tension flexible cable, but using the cable becomes difficult at accelerating voltages higher than 1 MV. This is one reason why the 3 and 3.5 MV ultraHVEMs at Osaka University were built with a tank-intank system, ${ }^{20), 58)}$ and the EMs themselves were suspended through cushions, as shown in Fig. $1{ }^{20)}$ Furthermore, mainly air cushions combined with rubber cushions were used for the mechanical vibration proofing of EMs. ${ }^{20)}$ As a result, the working time of the $3 \mathrm{MV}$ and the 3.5 MV ultra-HVEMs exceeds that of conventional 100 and $200 \mathrm{kV}$ EMs. A 5 MV ultra-HVEM was also designed when the 3.5 MV ultra-HVEM was being constructed. ${ }^{59)}$ In addition, the use of a remote-controlled system operated from a separate room, means that the $\mathrm{X}$-ray shielding for the EM column poses no problems, and operating a $3.5 \mathrm{MV}$ ultra-HVEM becomes possible even from overseas through a communication cable system. ${ }^{60), 61)}$

\section{References}

1) Bollmann, W. (1956) Phys. Rev. 103, 1588-1589.

2) Hirsch, P. B., Horne, R. W., and Whelan, M. J. (1956) Phil. Mag. 1, 677-684.

3) Nishiyama, Z., and Fujita, H. (1957) Metal Phys. 3, 108-110 (in Japanese)

4) Fujita, H., Taoka, T., and NRIM-500kV Group (1965) J. Electr. Microsc. 14, 307-308

5) Fujita, H. (1966) J. Phys. Soc. Jpn. 21, 1605

6) Fujita, H. (1966) Bull. Inst. Metal. Jpn. 5, 85-92 (in Japanese).

7) Fujita, H., Kawasaki, Y., Furubayashi, E., Kajiwara, S., and Taoka, T. (1967) Jpn. J. Appl. Phys. 6, 214-230.

8) Fujita, H. (1986) J. Electr. Microsc. Techn. 3, 243-304. 
9) Yamaguchi, K. (1929) Scientific papers of the Inst. of Phys. and Chem. Research 11, 224-245.

10) Heidenreich, R. D. (1949) J. Appl. Phys. 20, 993-1010.

11) Hirsch, P. B. (1962) J. Phys. Soc. Jpn. 17 (Suppl. B-II), 143-154.

12) Dupouy, G., and Perrier, F. (1966) Proc. 6th Int. Cong. Electr. Microsc., Kyoto 1, 107-108.

13) Uyeda, R., and Nonoyama, M. (1968) Jpn. J. Appl. Phys. 7, 200-208.

14) Hashimoto, H. (1971) Jernkont. Ann. 155, 479-490.

15) Kobayashi, K., and Ohara, M. (1966) Proc. 6th Int. Cong. Electr. Microsc., Kyoto 1, 579-580.

16) Shimadzu, S., Iwanaga, M., Kobayashi, K., Suito, E., Taoka, T., and Fujita, H. (1966) Proc. 6th Int. Cong. Electr. Microsc., Kyoto 1, 101-102.

17) Fujita, H. (1961) J. Phys. Soc. Jpn. 16, 397-406.

18) Uyeda, R. (1977) Proc. 5th Int. Conf. High Voltage Electr. Microsc., Kyoto, 3-8.

19) Fujita, H., Suzuki, K., and Sugata, E. (1970) Proc. 7th Int. Cong. Electr. Microsc., Grenoble 2, 329-330.

20) Ozasa, S., Kato, Y., Tadokoro, H., Kasai, S., Katagiri, S., Kimura, H., Sugata, E., Fukai, K., Fujita, H., and Ura, K. (1972) J. Electr. Microsc. 21, 109-118.

21) Fujita, H., and Tabata, T. (1973) Jpn. J. Appl. Phys. 12, 471-472 (received November 17, 1972).

22) Fujita, H., Sumida, N., and Tabata, T. (1973) J. Phys. Soc. Jpn. 35, 224-229 (received December 25, 1972).

23) Fujita, H., Ura, K., Mori, H., Yoshida, K., Komatsu, M., Sakata, T., Miyauchi, K., Matsui, I., and Katsuta, S. (1989) Proc. 40th anniversary, Jpn. Soc. Electr. Microsc. P-A-15, 193

24) Takaoka, A., Ura, K., Mori, H., Katsuta, T., Matsui, I., and Hayashi, S. (1997) J. Electr. Microsc. 46, 447-456.

25) Fujita, H., Tabata, T., Yoshida, K., Sumida, N., and Katagiri, S. (1972) Jpn. J. Appl. Phys. 11, 1522-1536.

26) Howie, A. (1966) Phil. Mag. 14, 223-237.

27) Fujita, H., Tabata, T., Sumida, N., and Yoshida, K. (1973) Proc. 3rd Int. Conf. HVEM, Oxford, 426-432.

28) Fujita, H. (1996) Mater. Trans. JIM 37, 1645-1654.

29) Humphreys, C. T., Thomas, L. E., Lally, J. S., and Fisher, R. M. (1971) Phil. Mag. 23, 87-114.

30) Fujimoto, F., Takagi, S., and Komaki, K., Koike, H., and Uchida, Y. (1972) Radiation Effects 12,153-161.

31) Fujita, H. (1989) J. Electr. Microsc. Techn. 12, 201-218.

32) Fujita, H., and Sumida, N. (1973) J. Phys. Soc. Jpn. 34, 838.

33) Imura, T., Saka, H., and Yukawa, N. (1969) Jpn. J. Appl. Phys. $\mathbf{8}, 405$.

34) Fujita, H. (1978) Proc. 9th Int. Cong. Electr. Microsc., Tronto III, 355-366.

35) Fujita, H., and Yamada, H. (1970) J. Phys. Soc. Jpn. 29, 132-139.

36) Fujita, H. (1990) Mater. Trans. JIM 31, 523-537.
37) Fujita, H., and Kimura, S. (1983) J. Phys. Soc. Jpn. 52, 157-167.

38) Fujita, H., and Yamada, H. (1968) Proc. Int. Conf. Strength of Metals and Alloys, Tokyo, 943-948.

39) Tabata, T., Mori, H., Fujita, H., and Ishikawa, I. (1976) J. Phys. Soc. Jpn. 40, 1103-1111.

40) Fujita, H. (1969) J. Phys. Soc. Jpn. 26, 1437-1445.

41) Fujita, H., and Miyazaki, S. (1978) Acta Metal. 26, 1273-1281.

42) Fujita, H., and Fujita, N. (2002) Radiation Effects and Defects in Solids 157, 85-100.

43) Fujita, H., and Yoshida, K. (1974) J. Phys. Soc. Jpn. 37, 986-993.

44) Mori, H., and Fujita, H. (1988) Proc. Int. Symp. on NonEquilibrium Solid Phases of Metals and Alloys 29, 93-96.

45) Sakata, T., Fujita, H., and Sumida, N. (1986) Proc. 11th Int. Cong. Electr. Microsc., Kyoto 2, 1545-1546.

46) Fujita, H., Komatsu, M., Sakata, T., and Fujita, N. (1996) Mater. Trans. JIM 37, 1350-1355.

47) Fujita, H., and Mori, H. (1988) Proc. Int. Symp. on NonEquilibrium Solid Phases of Metals and Alloys 29, 37-40.

48) Mori, H., Sakata, T., Komatsu, M., and Yasuda, H. (1993) Microsc. Microanal. Microstruct. 4,137-142.

49) Fujita, H. (1994) Mater. Trans. JIM 35, 563-575.

50) Fujita, H. (1986) J. Electr. Microsc. Techn. 3, 45-56.

51) Fujita, H. (1999) J. Electr. Microsc. 48 (Suppl.), 983-994.

52) Mori, H., Komatsu, M., Takeda, K., and Fujita, H. (1991) Phil. Mag. Lett. 63, 173-178.

53) Yasuda, H., Mori, H., Takeda, K., and Fujita, H. (1993) Proc. DIMAT-92, Defect and Diffusion Forum 95-98, 697-702.

54) Arata, Y., Fujita, H., and Zhang, Y. C. (2002) Proc. Jpn. Acad., Ser. B 78, 63-68.

55) Yasuda, H., and Mori, H. (1996) Z. Phys. D37, 181-186.

56) Fujita, H., and Sumida, N. (1986) Hitachi Sci. Instr. News 29, 2586.

57) Fujita, H., Taoka, T., and others of NRIM 500kVEM Group, Iwasa, N., and Kanaya, K. (1966) Appl. Phys. Lett. 8, 325-326.

58) Matsui, Y., Horiuchi, S., Bando, Y., Kitami, Y., Yokoyama, M., Suehara, S., Matsui, I., and Katsuta, T. (1991) Ultramicroscopy 39, 8-20.

59) Takaoka, K., Ura, K., and Fujita, H. (1986) Proc. 11th Int. Cong. on Electr. Microsc., Kyoto, 89-92.

60) Yoshida, K., Takaoka, A., Hayashi, S., and Matsui, I. (1999) J. Electr. Microsc. 48, 865-872.

61) Yoshida, K., Mori, H., Shimojo, S., Kadobayashi, Y., Akiyama, T., and Ellisman, M. H. (2002) J. Electr. Microsc. 51(Suppl.), S253-S257.

(Received April 12, 2005; accepted May 12, 2005) 


\section{Profile}

Dr. Hiroshi Fujita was born in 1926, and graduated in 1952 from the Department of Metallurgy, Faculty of Engineering, Osaka University. A special research assistant during his doctoral course, he thereafter moved to the Institute of Scientific and Industrial Research, Osaka University. Since that time, his research has been focused on the behavior of lattice defects in inorganic materials, especially metals and alloys. He has done pioneer work in Japan on the direct observation of dislocations with a $50 \mathrm{kV}$ electron microscope, and has discovered a new phenomenon called "subgrain grouping," which is the origin of nucleation during recrystallization. In 1963-67, he worked at the National Research Institute for Metals of the Science and Technology Agency in Tokyo, and performed pioneering work on the usefulness of HVEMs for materials science, despite the generally pessimistic views on this sub-

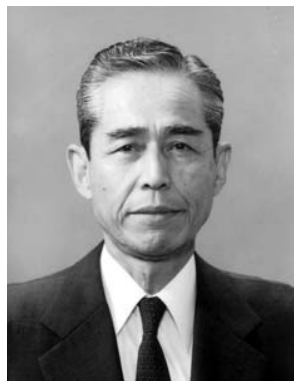
ject prevalent at that time, by constructing two practical 0.5 MV HVEMs in cooperation with

Shimadzu, Ltd. Thereafter, he became Professor at Osaka University in 1967, where he educated many students at the Department of Metallurgy in the field of physical metallurgy. At the same time, he established the Research Center for Ultra-high Voltage Electron Microscopy, constructing a 3 MV ultra-HVEM in 1970-72 in cooperation with Professor K. Ura and Hitachi, Ltd. Using both the HVEM and ultra-HVEM, he has shown the importance of electron channeling at high voltages, and has clarified by in-situ observation the mechanisms behind various phenomena representative of almost all bulk inorganic materials. Furthermore, he has also developed indispensable applications of the ultra-HVEM, using high-energy electrons in fields such as the implantation of foreign atoms into substrates, the induction of the crystallineto-amorphous transition in various materials, and the determination of the nucleus size of crystals, under which materials change their behavior anomalously as atom clusters. He has obtained the best data world-wide from transmission electron microscopes, producing the best image resolutions, accelerating voltages and functional features for various specimen-treatment devices used in in-situ observation, etc.

He has organized two International Conferences on HVEMs, has chaired the "Symposium on the formation of new functional materials" for more than 10 years, and has presided over the "Symposium on cluster science and technology" (currently the Society of Nano Science and Technology) for 4 years in Japan. He was awarded the Shiju Hosho Medal in 1993, the Japan Academy Prize in 1994, etc. He was elected a member of The Japan Academy in 2003. 